\title{
Simulating Wave Propagation Distribution Through GIS Integration
}

\author{
LUIS CARLOS MANRIQUE, ANTHONY WEISS, SANDRA PUENTES \\ Data scientist, Engineer, Assistant professor \\ Credit Engine Inc., Moneytree Inc., The University of Tsukuba \\ Tokyo Minato-ku Roppongi 7-15-7, Tokyo Minato-ku Castalia 2F, 3-13-3 Nishiazabu \\ Ibaraki Prefecture Tsukuba 1-1-1 Tennodai \\ JAPAN
}

\begin{abstract}
The use of electronic devices designed for user location estimation has become widely popular in the last decade. This is thanks to emergent technologies such as Bluetooth Low Energy, Radio-Frequency Identification, and Ultra-WideBand (UWB) among others. In the present study; the authors provide a method for using a Geographic Information System (GIS) to define spatial constraints, in order to simulate the lines of sight of anchors to make an informed selection of adequate locations for installation. By leveraging GIS, researchers or enterprises can improve the installation process by reducing costs while setting up arrangements that will ensure reliable data collection. We include a scenario illustrating the possibility of budget reduction of around $30 \%$ related to the orientation and survey of the devices.
\end{abstract}

Key-Words: Geographic Information Systems, Bluetooth Low Energy (BLE), indoor simulation, Ultra-Wideband (UWB), indoor location detection.

Received: January 19, 2021. Revised: May 30, 2021. Accepted: June17, 2021. Published: July 7, 2021.

\section{Introduction}

Tracking information collected from different objects as well as users has become a valuable resource for companies in multiple sectors. Regarding outdoor tracking, the Global Positioning System (GPS) is the most commonly used resource. However, for indoor tracking, the GPS doesn't work well since mechanical barriers such as roofs and walls may block or reflect signals. For this reason, other technologies must be used to identify an individual or product in a specific place at a specific time indoors.

In location-based services, devices such as Bluetooth Low Energy (BLE), Ultra-Wideband (UWB), and Radio Frequency Identification (RFID) tags provide tracking information by broadcasting or receiving signals that can be used to estimate the proximity of another device within a certain area. In an ideal situation, the devices should be installed in an open space with no interference. However, indoor structures as walls or large objects may generate shadows or blind spots where the system signal is unable to reach.

From the technology mentioned above, UWB is the most expensive. Therefore, finding a way to install the fewest UWB anchors while getting the maximum visibility possible will reduce costs and improve the data collection process as well.

\subsection{Indoor proximity services}

The placement of BLE beacons, RFID tags, UWB devices, and so forth in an indoor environment serves as absolute reference positions that can be used to calculate the location of pedestrians.

We will describe UWB very briefly here. Please refer to [1] for further details. UWB technology allows the mobility of wireless communications at high speeds. These devices are specified to have a $-10 \mathrm{~dB}$ fractional bandwidth greater than $20 \%$ or at least $500 \mathrm{MHz}$ of the occupancy spectrum [2]. The US Federal Communications Commission (FCC) specifies that the power emission levels of UWB must be within the spectrum of $3.1-10.6$ $\mathrm{GHz}$ to assure coexistence with other signals in the same band [1].

UWB is nowadays one of the most accurate indoor systems. The accuracy is within centimeters [3]. However, some smoothing or correcting methods such as a Kalman filter or Loess regression may be applied to reduce the noise in the estimated path $[4]$.

\subsection{Related work}

Different methods have been studied for optimizing the positioning and reducing the number of used UWB anchors. Genetic algorithms have been a popular approach; Barret [5] studied the problem by simulating the sensor readings while using a probabilistic model where the chance of detection was directly proportional to $1 /$ Range $^{2}$. The study attempted to place sensors in the Hudson River in a random way to detect intruders. The algorithm found the Pareto frontier and the curve of optimal trade-offs between the objectives. Olague and Mohr [6] optimized the placement of cameras 
to minimize the error in sensing. They analyzed the problem in two steps; in the first step, they chose a criterion with the maximum element in the diagonal of the covariance matrix which depends on the error propagation. And in the second one, they created a heuristic to minimize the previous criterion. Xia and collaborators [7] measured the distance from the tag to the base stations and proposed a positioning equation that is solved by the genetic algorithm. The authors realized the importance of considering other variables rather than only the line of sight.

Monica and Ferrari [8] introduced an analytical approach to optimize anchor nodes placement using time- difference-of-arrival UWB-based localization of a moving target node. The authors developed a deeper analysis based on the hypothesis that the target will move in a straight line in the middle of a corridor. Other methods of positioning have been studied by using Taylor series expansion [9] and Levenberg-Marquardt algorithm [10]. Particularly for improvement of indoors positioning, some papers have been introduced such as the one by Hol and colleagues implementing UWB calibration [11] and the study by Ridolfi et al., using particle filters as well as Kalman filter to estimate the localization of UWB for analyzing sports postures [12]. The study by Arsan et al. analyzed behavior mapping (BM) by using three different technologies Wi-Fi, BLE Beacon, and UWB sensors. They found the best performance by using the UWB sensors for BM [13]. Suwatthikul et al experimented by comparing the performance of a UWB system in different scenarios. They demonstrated that configuring the UWB with the maximum range did not outperform when there was a clear communication path [14].

Regarding GIS, ArcGIS indoors appears to be an interesting approach. Introduced by ESRI; one of the largest companies that work on GIS, this tool allows to place different spatial features such as layouts, sensors, routes, among others in a simulated space. However, it does not have features for simulating signals in indoor locations. To our knowledge, there are no investigations related to the integration of GIS and signal broadcasting.

For the present study, we propose a virtual approach based on simulations of the physical properties of a space designed for placing UWB anchors, followed by a simulation of the anchors' signal propagation to support the setup process.

Additionally, we added to the simulation, the possibility to be transferable to different locations. In order to approach this issue, we explored the possibility of implementing a Coordinate Reference System (CRS). The Geographic Information
System (GIS) was useful for gathering and displaying geographic information. This system structures and gives format to the spatial information by using a CRS, a set of rules to identify points in the real world as data[15]. Furthermore, it can also be integrated with economic, demographic, social, or mobility data. The relevance of this investigation relies upon the integration of GIS with signal propagations in indoor environments. The implementation of such simulations may provide companies and researchers with useful information for anchor positioning and its optimal distribution indoors, also providing the ability to estimate and minimize costs while gaining the best distribution for the acquisition of quality data. Although we propose the implementation of UWB for the present paper, we consider that the same analysis may be also usable for BLE beacons positioning, Received Signal Strength Indicators (RSSI) evaluation, among other technologies.

\section{Materials and Methods 2.1 Geographic Information System (GIS)}

A GIS combines hardware and software to manipulate, store, retrieve, view, and analyze large spatial databases [16][17]. For this study, we will use QGIS, since it is freeware and can be connected to other software and tools straightforwardly. QGIS is an official project of the Open Source Geospatial Foundation (OSGeo). It runs on Linux, Unix, MacOS, Windows, and Android, and supports numerous vector, raster, and database formats and functionalities $18 \|$.

QGIS allows the user to connect the software with other programs through a toolbox named "processing." It also contains several algorithms grouped in different blocks, and allows running single or batch processes. By using spatial functions, new objects can be imported into QGIS. The toolbox can be downloaded and integrated into QGIS by installing the plugin called "Processing R provider".

A screenshot of the processing toolbox is shown in figure 1. For this study, we will connect QGIS 3.16 with Microsoft R Open 4.0.2 to create spatial features with a CRS by running a batch file. $R$ was used because of the complexity of calculations.

There are many CRSs such as the Universal Transverse Mercator (UTM), World Geodetic System 84 (WGS 84), a global standard for latitude and longitude positions, among others. For this study, the authors will use the World Mercator: 54004 CRS using meters instead of angles to locate the spatial features relatively. Later they can be projected if required. 


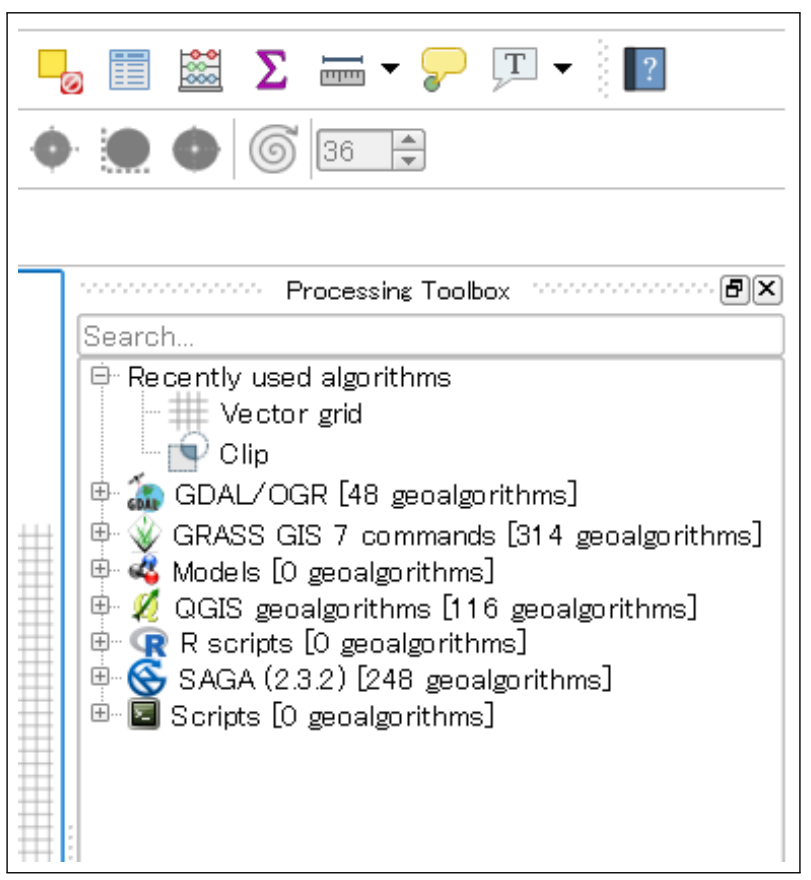

Figure 1: QGIS Processing Toolbox. Through this toolbox, QGIS allows to connect the software with other programs easily.

\subsection{Simulations through $R$ software}

$\mathrm{R}$ is 'GNU S,' a freely available language and environment for statistical computing and graphics which provides a wide variety of statistical and graphical techniques: linear and nonlinear modeling, statistical tests, time-series analysis, classification, clustering, among others. [19]

The libraries used in the current manuscript are: maptools, sp, rgeos and rgdal. They are available for free on the R-project website.

The spatial files were generated from $\mathrm{R}$, then read and imported into QGIS. A special characteristic of the simulation provided and tested by the authors is that the CRS of the layout (walls, columns, and other polygons) must be the same as the generated tag to generate the object at the desired place.

\subsection{Methods \\ 2.3.1 Path loss identification}

The radio signals broadcast by the UWB tag are emitted through space and are affected by spatial restrictions such as walls, doors, or windows, among others. These restrictions reduce the power of the wave in the space; this phenomenon is called 'path loss'.

The path loss for UWB devices can be described as follows:

$$
\operatorname{PathLoss}(d)=C_{0}+10 n \log _{10}\left(\frac{\lambda}{4 * \pi * d}\right)+x_{\sigma}
$$

Where the path loss is given in decibels $(\mathrm{dB})$. $C_{0}$ is the loss at the reference distance of 1 meter. $\lambda$ is the center of the wavelength in meters. $d$ is the distance in meters. $x_{\sigma}$ is the shadow fading and $n$ is the path loss exponent, equal to 2, for open spaces [20].

UWBs are used for positioning by using the time difference from arrival, angle of arrival, time of arrival, received signal strength indication among others. Some of them are explained as follows:

a. Time difference from arrival (TDOA): Measures the time difference between the UWB signal from the tag to multiple UWB anchors. This calculation allows the system to identify the location of a moving tag. Since the power consumption of the tag is low, it is easier to identify several tags.

b. Angle of arrival (AOA): The position of the tag is calculated by using the signal incidence angle to the anchors. When the whole system includes multiple anchors, the angle of arrival is calculated by using a minimum of 3 of them.

c. Time of arrival (TOA): The position of the tag is calculated by recording the time it takes from the tag to more than 3 anchors. The system then estimates the location of the tag by using the distances from the tag to each anchor through the triangulation principle. The tags, as well as the anchors, must be synchronized to avoid problems.

d. Received signal strength indication: This application is similar to the BLEs and Wi-Fi. It uses the RSSI value to estimate the location of the tag. However, methods such as TOA provide better accuracy given that the location is estimated by the pulse of the signal.

\subsubsection{Broadcasting signals simulation on GIS}

To simulate the signal propagation based on spatial constraints and distance from the origin, a layout was created using a GIS. For generating the coverage area of the UWB anchor, the following parameters were defined before generating the spatial feature:

a. Walls: spatial constraints such as scaffolds, columns, rooms, and any physical object that might interfere with the signal propagation.

b. nSegments: The total number of dots that will be generated between the origin of the signal and the defined propagated distance. 


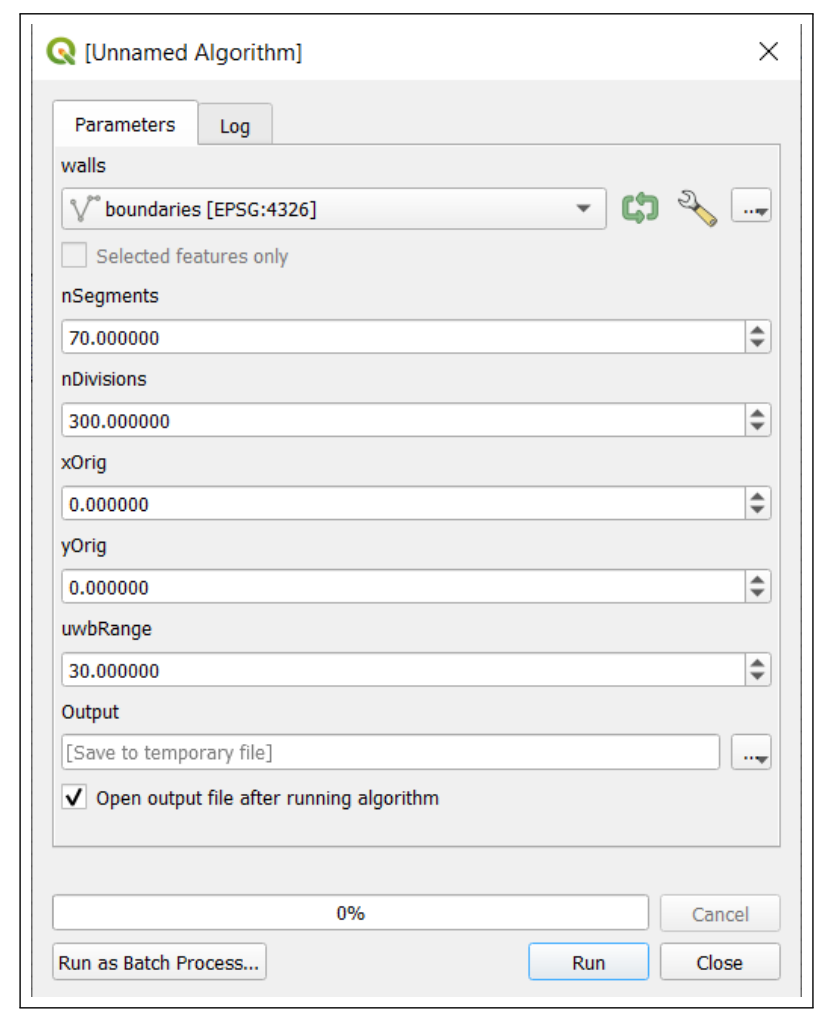

Figure 2: Tool for simulating wave propagation.

c. nDivisions: Represents the number of "rays" that will be emitted in total. This value will provide the angle between rays, it is defined as: angle $=n$ Divisions $/ 360$.

d. xOrig: Location at $x$.

e. yOrig: Location at $y$.

f. uwbRange: The distance where the UWB can get accurate data. In theory, it can be between 45 to $60 \mathrm{~m}$ from the origin.

The used tool also allows changing the parameters regarding the conditions of the proposed simulation A snapshot of this feature can be seen in figure 2 .

\subsubsection{Simulated environment}

After broadcasting the signal simulation, a simulated mesh layout was obtained. Figure 3 shows an example where each mesh area corresponds to one square meter and the black squares distributed homogeneously through the plane represent the columns in the area. The polygons with diagonal red lines correspond to scaffolds, shelves, or other physical obstacles.

The algorithm uses the parameters described in section 2.3.2 to simulate the area coverage from a UWB anchor until some distance where the signal is no longer reliable. Next, we overlaid the

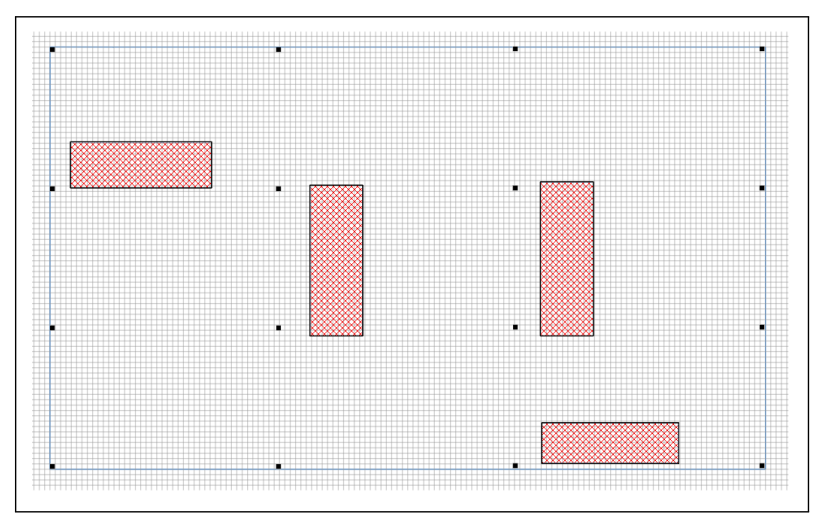

Figure 3: Simulated environment: The black squares making a reticle represent the columns in the area. The polygons with diagonal red lines correspond to scaffolds, shelves, or other physical obstacles.

propagated points with the GIS diagram. From each line of sight, the algorithm identifies if the signal is blocked by a polygon and assigns a specific value at a specific point. Since this process uses geographical features, it is possible to add even more information to $x$ and $y$ coordinates. In this case, we add information related to obstacles that are overlaying or blocking the line of sight.

Once the process is finished, we define the shadow as follows:

$$
\text { startingShadow }_{i a}=\min \left(\text { lineSight }_{i a} \neq 0\right)
$$

where $i$ refers to the line of sight $i$, and $a$ refers to the position where a polygon is interfering with the line of sight $i$.

Later, we iterated through all the lines of sight as follows.

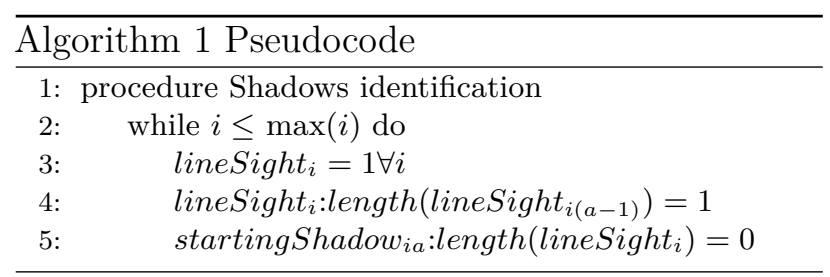

Regarding details of the algorithm 1:

- Line 3: Define the status of all points in a line of sight $i$ as 1 . The initial assumption was that there was no obstruction.

- Line 4: Once a point $a$ is overlaid with a physical constraint, it changes the status of that point to 0 . It means that a shadow starts at the point $a$. 


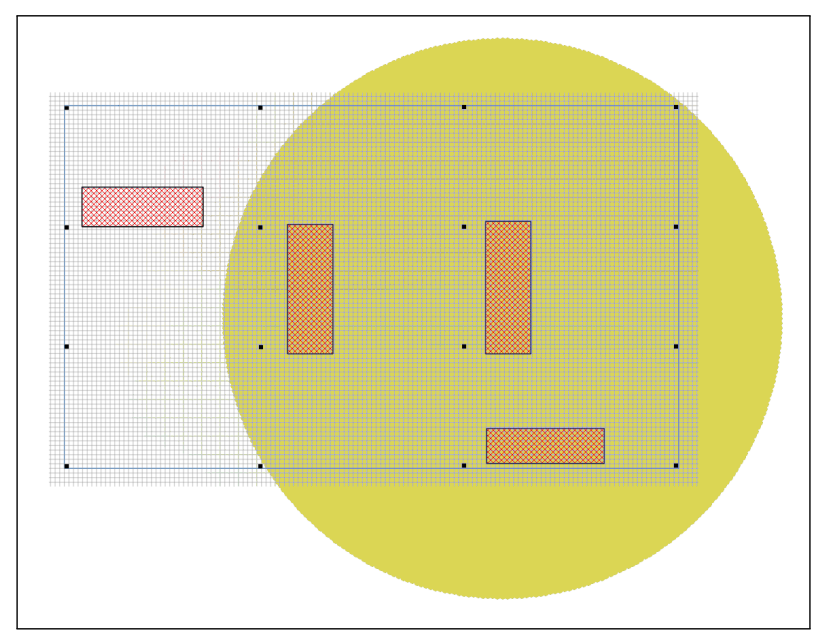

Figure 4: Generated signal: The radius of coverage of the UWB anchor is shown by the yellow circle.

- Line 5: Define the status of the subsequent points in the line of sight $i$ as 0 .

We applied this process to all the lines of sight to test if they were obstructed by physical constraints. Given that the UWB antenna will be moving given specific activities such as tracking raw materials, tracking staff, etc., the interference with objects will reduce the positioning accuracy [22].

\section{Results}

\subsection{GIS and signal simulation}

In Figure 4, the signal propagation from certain coordinates during a simulation is displayed. We used the radius that a UWB can broadcast with high accuracy $(60 \mathrm{~m})$.

In Figure 5, a detailed diagram of the simulated signal is shown. The black squares represent columns, and the polygon in the center represents an obstruction such as a shelf.

Figure 6 shows that it is possible to see the simulated signal in two dimensions. The red area represents the direction that the UWB is not broadcasting. The line of sight is detailed in green, and the areas not covered by the signal due to obstruction by physical objects are generated in blue, columns and scaffolds are also shown.

By integrating GIS it is easier to calculate the effective area coverage for the UWB anchors considering all the parameters included in the tool for broadcasting signals. This calculation may help genetic algorithms or other methods for optimization to deal with multiple objective functions such as maximize the area coverage and reducing the number of UWB anchors.

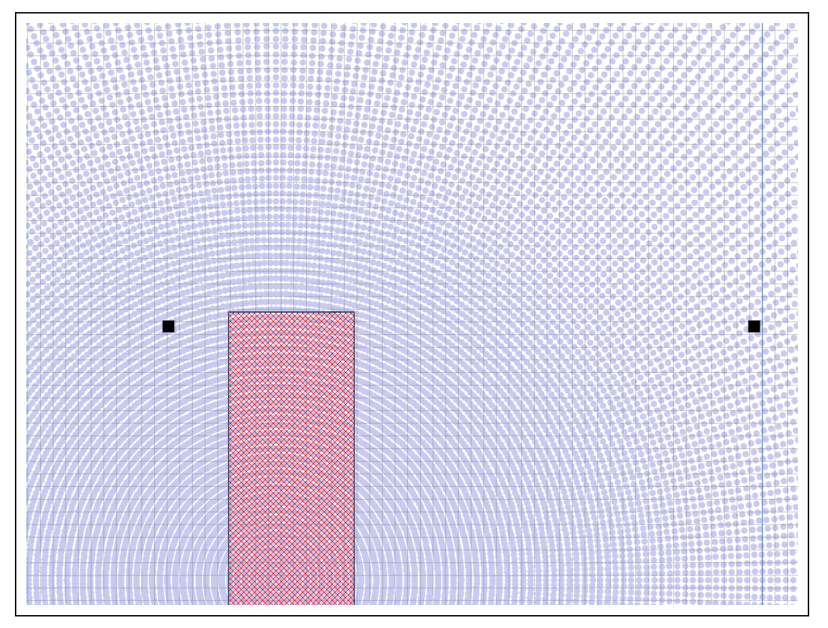

Figure 5: Detail of Shapefile: Spatial points are generated by modifying parameters included in the tool.

\subsection{Costs}

The deployment of indoor location tracking systems generates costs including the configuration and installation, support and maintenance, monthly fees, loss, and firmware updates, among others.

One of the most challenging steps during the implementation is the setup of the devices due to the small tag antenna location and the interaction with the physical environment. Ideally, the anchors should be placed appropriately, since changing their position brings other costs related to cable extension and re-positioning [23]. In the investigation conducted by Wang and Pan, it was evident the difficulties of placing the anchors by using a total station or laser range finder. Given that in most of the cases the antenna will move, other systems such as Light Detection and Ranging (LiDAR) could be implemented to calibrate the system to reduce the errors and thus improve the accuracy [24].

The price of buying 5 Ubisense UWB compact tags, suitable for mounting into objects and vehicles is about 18,500 USD (2,000,000 JPY). Other devices, such as the "Ubisense series 9000 IP sensor" that consists of an array antenna and an ultraUWB RF receiver of $4 \times 4 \times 1.7 \mathrm{~cm}$ are useful for mounting on people or objects[25]. Just 4 sensors cover an area of $200 \mathrm{~m}^{2}$ [26].

If a company wants to cover an area of about $10,570 \mathrm{~m}^{2}$ (as the simulated area used for this study), 22 sets of sensors are required. If they test the installation for 3 weeks, the total cost per hour is about $\$ 2,775$ USD. The average time spent for experienced workers with this technology could be around 22 hours, this covers bipods place- 

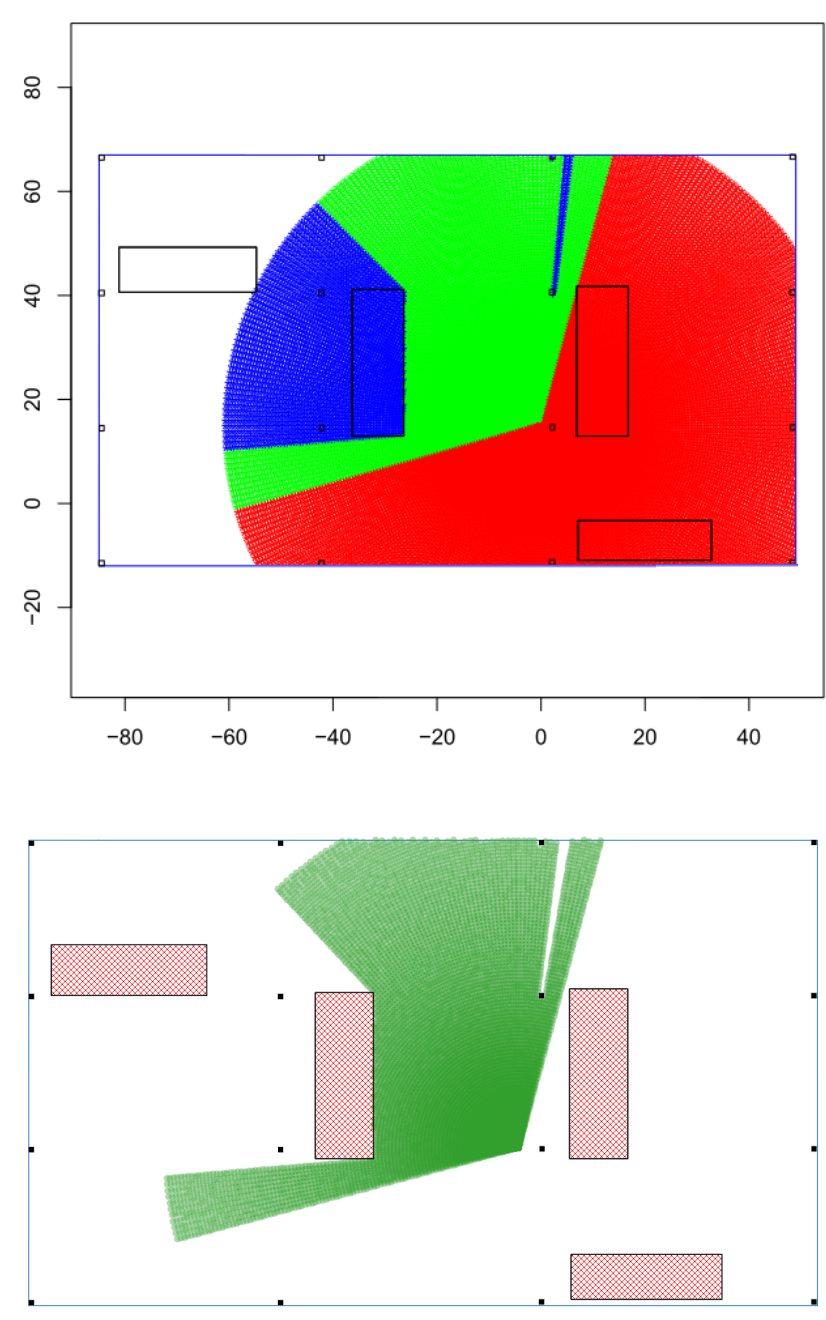

Figure 6: Projecting shadows and line of sight: The upper figure shows shadows in blue, line of sight in green and non-visible area in red. The coordinates are given in meters. The lower figure shows the line of sight in green with an effective area coverage of $2,288 \mathrm{~m}^{2}$.

ment, sensor placements, layout and cabling, orientation and survey, and system calibration. Following Umer et al, the total time related to the orientation and survey is about $27 \%-34 \%$ of the total time of the deployment [27]. Translated into man-hours, this is about 8 to 10 hours. By using our proposed method, it is possible to reduce this time to less than $2-3$ hours since the setup is determined beforehand via simulations.

\section{Discussion}

One of the most difficult decisions for setting up broadcast devices is how to place them for maximum effectiveness and cost reduction. Some algorithms have been proposed for this problem. How- ever, not all companies or industries are familiar with them. Thus, a simulation in situ could be easier to implement and help reducing setup costs, since users will be able to see how the signal is propagated.

Genetic algorithms have been applied to optimize the number and placement of the anchors indoors. However, they have some disadvantages like the formulation of the fitness function, the parameters, the rate of mutation and crossover and the selection criteria of the new population [28]. One of the main issues is that the algorithm may not converge to an optimal solution or global optima [29]. Also, most of the applications on this topic have not considered spatial constraints that affect the optimization process. The investigations have been conducted in open spaces or clear corridors without considering physical limitations.

Renting UWB equipment is a cost that a company should consider because this technology can achieve high accuracy, but it requires an important economic investment. Other technologies such as BLE may be cheaper, but the area of coverage is smaller, accounting just for a few meters from the BLE placement in contrast to the coverage provided by a UWB anchor which may cover about $60 \mathrm{~m}$ (or an area of $11,310 \mathrm{~m}^{2}$ ). If a company or researchers want to define an effective area where the RSSI is bigger than $-60 \mathrm{dBm}$, it means that the BLE beacon will cover just a range of about $2.5 \mathrm{~m}$ [30].

As for limitations, the 2-dimensional analysis does not account for all the spatial features that can exist in an indoor environment. If the device is in a high place (usual condition), our approach cannot fully represent how the signal is obstructed. However, this proposal does allow the workers and researchers to have an idea of the signal behavior before implementation.

Further, it is possible to include additional information inside each feature of the shapefiles, such as signal loss, type of obstruction, among others. This also helps to estimate and conduct a kernel density method in two dimensions by using the signal properties. For the spatial constraints such as walls, doors, etc., it is possible to integrate the information related to the obstruction to estimate the signal loss or power at some point $(\mathrm{x}, \mathrm{y})$.

\section{Conclusion}

The tool introduced in this document can be defined using coordinate reference systems. By implementing this tool, companies, as well as researchers, will be able to estimate signal propagation in specific indoor environments. This feature may be helpful to control several parameters 
to get the required information and thus perform simulations related to the location of the devices.

Another advantage of this tool is that by integrating GIS, it would be possible to incorporate it with different algorithms towards an optimization model. Finding the best parameters for the different variables will help companies and researchers to place the UWB anchors in the best way to minimize the number of tests before the experiments.

Our tool considers spatial features loaded in GIS as range and location. So, it is possible to use it for simulating other types of technologies such as BLE beacons or Wi-Fi Antennas as well. Also, since the tool projects several spatial points from an origin, more properties can be given to each of them. Properties such as the strength of a radio signal in a clear environment or its attenuation across materials [31].

While enterprise systems such as TruNET Wireless can accomplish similar results, a commercial license is required. Another reason the authors worked with R and Q-GIS specifically is that these tools allow companies to reduce costs since no commercial software or license is required for its implementation.

Finally, this paper contributes to electronic devices given the advantages of integrating not only spatial features into the signal broadcast but also other intrinsic properties such as power strength (RSSI), power loss, among others; and the parameters of the spatial features can be adjusted under the optimization models.

\section{References:}

[1] Rahayu, Yusnita and Abd Rahman, et al., 2008 5th IFIP International Conference on Wireless and Optical Communications Networks (WOCN '08), doi: 10.1109/WOCN.2008.4542537, 2008.

[2] Nguyen, C. and Miao, M., Fundamentals of UWB Impulse Systems. Design of CMOS RFIC Ultra-Wideband Impulse Transmitters and Receivers, Springer International Publishing, 2017.

[3] Abdulrahman Alarifi and AbdulMalik S and et al, Ultra Wideband Indoor Positioning Technologies: Analysis and Recent Advances, Sensors (Basel), doi: 10.3390/s16050707, 2016.

[4] Gabriel-Adrian Deak and Kevin Curran and Joan Condell, Filters for RSSI-based measurements in a Device-free Passive Localisation Scenario, Image Processing and Communications, Vol.15, No.1, 2010, pp. 23-34.

[5] Barrett, S., Optimizing Sensor Placement for Intruder Detection with Genetic Al- gorithms, IEEE Intelligence and Security Informatics, 2007, pp. 185-188, doi: 10.1109/ISI.2007.379555.

[6] Olague and Mohr, Optimal 3D Sensors Placement to Obtain Accurate 3D Points Positions Acressed. Inne 6 2021., <htt.n://citeseerx.ist.psu.edu/viewdoc/ summary?doi=10.1.1.56.2375 >

[7] Xia, Bin, et al., UWB Positioning System Based on Genetic Algorithm., Journal of Computer and Communications, Vol. 9, Issue 04, 2021, pp 110-118.

[8] Monica, S. and Ferrari, G., UWB-based localization in large indoor scenarios: optimized placement of anchor nodes, IEEE Transactions on Aerospace and Electronic Systems, Vol.51, No.2, 2015, pp. 987-999, 10.1109/TAES.2014.130722.

[9] Foy, W., Position-Location Solutions by Taylor-Series Estimation, IEEE Transactions on Aerospace and Electronic Systems, vol. AES-12, no. 2, 1976, pp. 187-194, doi: 10.1109/TAES.1976.308294.

[10] Mensing, C. and Plass, S., Positioning Algorithms for Cellular Networks Using TDOA, IEEE International Conference on Acoustics Speech and Signal Processing Proceedings, 2006, pp. IV-IV, doi: 10.1109/ICASSP.2006.1661018.

[11] Hol, J., Schön, T., and Gustafsson, F., Ultra-wideband calibration for indoor positioning, IEEE International Conference on Ultra-Wideband, 2010, pp. 1-4, doi: 10.1109/ICUWB.2010.5616867.

[12] Ridolfi, M., Vandermeeren, S., et al. Experimental Evaluation of UWB Indoor Positioning for Sport Postures Sensors, Vol. 18, No 168, 2018, pp. 1-20, <https://doi.org/10. 3390/s18010168>.

[13] Arsan, T., Kepez, O., Early Steps in Automated Behavior Mapping via Indoor Sensors. Sensors, Vol. 17, No. 2925, 2017, <https: //doi.org/10.3390/s17122925>.

[14] Suwatthikul, C., Chantaweesomboon, W., Manatrinon,S., Athikulwongse, K. and Kaemarungsi, K., Implication of anchor placement on performance of UWB real-time locating system, 8th International Conference of Information and Communication Technology for Embedded Systems (IC-ICTES), 2017, pp. 1-6, doi: 10.1109/ICTEmSys.2017.7958760.

[15] Parker, A., The South African coordinate reference system (part 1), PositionIT, Nov/Dec, 2011. 
[16] Berling-Wolff, Sheryl and Wu, Jianguo. Modeling urban landscape dynamics: A review. Ecological Research, Vol.19, No.1, 2004, pp. 119-129.

[17] Manrique, Luis Carlos and Yamamoto, Kayoko., Support vector machine for land use through socio-economic factors applied to a compact city model, Recent Advances in $\mathrm{Au}-$ tomatic Control, Modelling and Simulation, Vol.7, No.11, 2013, pp. 113-120.

[18] QGIS Development Team. QGIS Geographic Information System. Open Snurce Genspatial Foundation, 2009, <http://qgis.osgeo.org $>$.

[19] R Core Team, R: A Language and Environment for Statistical Computing, R Foundation for Statistical Computing, 2017.

[20] Rusch, Leslie and Prettie, et al, Characterization of UWB Propagation from 2 to $8 \mathrm{GHz}$ in a Residential Environment, IEEE Journal on Selected Areas in Communications - JSAC, 2003.

[21] Tarrío, Paula and Bernardos, Ana M. and Casar, José R., Weighted Least Squares Techniques for Improved Received Signal Strength Based Localization, Sensors, Vol.11, No.9, 2011, pp. 8569-8592.

[22] Ren A, Zhou F, Rahman A, et al., A study of indoor positioning based on UWB basestation configurations, IEEE 2nd Advanced Information Technology, Electronic and Automation Control Conference (IAEAC). IEEE, 2017, 1939-1943.

[23] Lubomir Mraz, 2019, Accuracy Considerations for UWB Indoor Tracking in an Industrial Environment, Accessed: April 24, 2021., <https://www.sewio.net/accuracyconsiderations-for-uwb-indoor-tracking-in-anindustrial-environment>.

[24] Wang, J. and Pan, R., Research and Evaluation of the Precision and Accuracy of UWB technology utilization in the Indoor Positioning Solution, Accessed: June 5, 2021., $<$ https://geo-matching.com/content/researchand-evaluation-of-the-precision-and-accuracyof-uwb-technology-utilization-in-the-indoorpositioning-solution $>$.

[25] Ubisense, a kit for verifying a positioning system using UWB hand radin. Accessed: A pril 24, 2021, <httns:/ /active nikkeihn co. jp/atclact/active/17/071100318/101800575>.

[26] Elkhouly, Essam Abdelkadir, UWB Precise Indoor Localization System
Performance, Limitations and its Integration. PhD diss., University of Tennessee, 2014. Accessed: April 26, 2021., $<$ https://trace.tennessee.edu/utk_graddiss $/ 3121>$.

[27] Umer, Waleed, and Mohsin K Siddiqui, Use of Ultra Wide Band Real-Time Location System on Construction Jobsites: Feasibility Study and Deployment Alternatives, International journal of environmental research and public health, vol. 17, No.7, 2020, doi:10.3390/ijerph17072219.

[28] Yang, X., Chapter 6 - Genetic Algorithms, Nature-Inspired Optimization Algorithms (Second Edition), pp.91-100, 2021, Academic Press, Second Edition.

[29] Mokhatab, S., Mak, J., Valappil, J., Wood, D., Chapter 5 - Natural Gas Liquefaction Cycle Enhancements and Optimization, Handbook of Liquefied Natural Gas, pp. 229-257, 2014, Gulf Professional Publishing.

[30] BLE Beacons for Indoor positioning - Beacon limitations Acressed. June 7, $\quad 2021, \quad<$ ht.tns://lncatify com/blog/ ble-beacons-no-bull-beacon-review/ $>$.

[31] The basics of signal attenuation. Cas-Data Lnogers Arressed. June 7 , 2021, <httns://www. datalnggerinc.com/ wp-content/1uploads/2016/11/16_Basics_of_ signal_attenuation.pdf $>$.

\section{Contribution of individual authors to the creation of a scientific article (ghostwriting policy)}

Luis Carlos Manrique carried out the writing of this manuscript, research and simulations.

Anthony Weiss carried out the writing and checking the logic of this manuscript.

Sandra Puentes carried out the writing of this manuscript.

\section{Sources of funding for research presented in a scientific article or scientific article itself}

Personal funding. 


\section{Creative Commons Attribution License 4.0 (Attribution 4.0 International, CC BY 4.0)}

This article is published under the terms of the Creative Commons Attribution License 4.0

https://creativecommons.org/licenses/by/4.0/deed.en_US 